\title{
Strange Stars in the Color-Flavor Locked Phase
}

\author{
Manuel Malaver ${ }^{1,2, *}$, Hamed Daei Kasmaei ${ }^{3}$ \\ ${ }^{1}$ Bijective Physics Institute, Slovenia \\ ${ }^{2}$ Department of Basic Sciences, Maritime University of the Caribbean, Venezuela \\ ${ }^{3}$ Department of Applied Mathematics and Computer Science, Islamic Azad University, Iran
}

Received November 3, 2019; Revised December 25, 2019; Accepted January 10, 2020

Copyright $\odot 2020$ by authors, all rights reserved. Authors agree that this article remains permanently open access under the terms of the Creative Commons Attribution License 4.0 International License

\begin{abstract}
We found new classes of exact models to the Einstein-Maxwell system of equations which describe the internal structure of a compact star made of strange matter considering the equation of state proposed by Rocha, Bernardo, de Avellar and Horvath in 2019. It has been assumed that this matter is composed of equal number of up, down and strange quarks and a small amount of electrons required to reaching the charge neutrality. If this hypothesis is correct, the neutron stars would be strange stars. We have chosen a particular form of gravitational potential $Z(x)$ that depends on an adjustable parameter related to degree of anisotropy of the models and the new solutions can be written in terms of elementary and polynomial functions. The obtained models satisfy all physical features expected in a realistic star and the expressions for mass, density and stellar radius are comparable with the experimental results.
\end{abstract}

Keywords Charge Neutrality, Gravitational Potential, Strange Stars, Anisotropy, Adjustable Parameter

\section{Introduction}

The analysis and description of gravitational collapse in ultracompacts objects has high importance in astrophysics and has attracted and influenced many physicists due to formulation of the general theory of relativity $[1,2]$. One of the most fundamental problems in theoretical physics is finding exact solutions of the Einstein field equations [3]. The exact solutions as physical model of compact stars was studied by Delgaty and Lake [4] who constructed several analytical solutions that describe static spherically symmetric perfect fluid and it satisfies all the necessary conditions to be physically acceptable and interesting topic as a case research.

In the construction of models of compact stars, the researches of Schwarzschild [5], Tolman [6] and Oppenheimer and Volkoff [7] are very important.
Schwarzschild [5] found analytical solutions that allowed describing a star with uniform density, Tolman [6] developed a method to find solutions of static spheres of fluid and Oppenheimer and Volkoff [7] used Tolman's solutions to study the gravitational balance of neutron stars. It is important to mention Chandrasekhar's contributions [8] in the model production of white dwarfs in presence of relativistic effects and the works of Baade and Zwicky [9] who propose the concept of neutron stars and identify an astronomic dense objects known as supernovas.

The description of the gravitational collapse and evolution of the compact objects has been a topic of great importance in general relativity. Recent experimental results in binary pulsars suggest that some compact objects could be quark stars [10]. The existence of quark stars in hydrostatic equilibrium was first suggested by Itoh [11] in a seminal treatment. The study of strange stars with quark matter has been a topic of great interest in the last decades since this could represent the most energetically favorable state of baryon matter [12].

Stellar models consisting of spherically symmetric distribution of matter with presence of anisotropy in the pressure have been widely considered in the frame of general relativity [13-25]. The existence of anisotropy within a star can be explained by the presence of a solid core, phase transitions, a type III super fluid, a pion condensation [26] or another physical phenomenon by the presence of an electrical field [27]. Many researchers and scientists have used a vast and great variety of mathematical techniques to try and test in order to obtain solutions of the Einstein-Maxwell field equations for anisotropic relativistic stars since it has been demonstrated by Komathiraj and Maharaj [28], Thirukkanesh and Maharaj [29], Maharaj et al.[30], Thirukkanesh and Ragel [31,32], Feroze and Siddiqui [33,34], Sunzu et al.[35], Pant et al. [36] and Malaver [37-40]. These analyses indicate that the system of Einstein-Maxwell equations is very important in the description of ultracompacts objects.

In order to analytically integrate field equations, the 
choice of the appropriate equation of state allows obtaining models of compact stars to be physically acceptable [41]. Komathiraj and Maharaj [12], Malaver [42], Bombaci [43], Thirukkanesh and Maharaj [29], Dey et al [44] and Usov [27] assume linear equation of state for quark stars. Feroze and Siddiqui [33] consider a quadratic equation of state for the matter distribution and specify particular forms for the gravitational potential and electric field intensity. Mafa Takisa and Maharaj [45] obtained new exact solutions to the Einstein-Maxwell system of equations with a polytropic equation of state. Thirukkanesh and Ragel [10] have obtained particular models of anisotropic fluids with polytropic equation of state, which are consistent with the reported experimental observations. Malaver [46] generated new exact solutions to the Einstein-Maxwell system considering Van der Waals modified equation of state with polytropic exponent. More recently, Rocha et al.[41] presented a new model with anisotropic pressure and an equation of state that describes the internal structure of a compact star made of strange matter in the color flavor locked (CFL) phase. This matter is assumed to be composed of equal numbers of up, down and strange quarks and a small number of electrons needed to maintain the charge neutrality. If this hypothesis is correct, neutron stars could be strange stars or hybrid stars with a thin crust of nuclei.

In this paper, we generated a new class of anisotropic matter with CFL matter equation of state proposed for Rocha et al.[41] in a static spherically symmetric space-time using a gravitational potential $Z(x)$ which depends on an adjustable parameter $\eta$. We obtained some new class of static spherically symmetrical models for an uncharged anisotropic matter distribution where the variation of the parameter modifies the radial pressure, energy density, stellar radius and the mass of the compact objects. This article is organized as follows: In Section 2, we present Einstein's field equations. In Section 3, we make a particular choice of gravitational potential $\mathrm{Z}(\mathrm{x})$ that allows solving the field equations and we have obtained new models for uncharged anisotropic matter. In Section 4, physical acceptability conditions are discussed. In section 5, a physical analysis of the new solutions is performed. Finally, in Section 6, we make a conclusion about obtained and discussed results.

\section{The Einstein-Maxwell Field Equations}

Consider a spherically symmetric four-dimensional space-time so that whose line element is given in Schwarzschild coordinates by

$$
d s^{2}=-e^{2 v(r)} d t^{2}+e^{2 \lambda(r)} d r^{2}+r^{2}\left(d \theta^{2}+\sin ^{2} \theta d \varphi^{2}\right)
$$

where $v(r)$ and $\lambda(r)$ are the two arbitrary functions. For uncharged anisotropic fluids, the Einstein-Maxwell system of field equations are obtained as follows:

$$
\begin{aligned}
& \frac{1}{r^{2}}\left(1-e^{-2 \lambda}\right)+\frac{2 \lambda^{\prime}}{r} e^{-2 \lambda}=\rho \\
& -\frac{1}{r^{2}}\left(1-e^{-2 \lambda}\right)+\frac{2 v^{\prime}}{r} e^{-2 \lambda}=p_{r} \\
& e^{-2 \lambda}\left(v^{\prime \prime}+v^{\prime 2}+\frac{v^{\prime}}{r}-v^{\prime} \lambda^{\prime}-\frac{\lambda^{\prime}}{r}\right)=p_{t}
\end{aligned}
$$

where $\rho$ is the energy density, $p_{r}$ is the radial pressure and $p_{t}$ is the tangential pressure, $\Delta$ is the anisotropy and primes denote differentiations with respect to $r$. Using the transformations suggested by Dugapal and Bannerji [47] as $x=c r^{2}, \quad Z(x)=e^{-2 \lambda(r)}$ and $A^{2} y^{2}(x)=e^{2 v(r)}$ where $\mathrm{A}$ and $\mathrm{c}$ are arbitrary constants, then the Einstein-Maxwell system has the equivalent form as follows :

$$
\begin{gathered}
\frac{1-Z}{x}-2 \dot{Z}=\frac{\rho}{c} \\
4 Z \frac{\dot{y}}{y}-\frac{1-Z}{x}=\frac{p_{r}}{c} \\
4 x Z \frac{\ddot{y}}{y}+(4 Z+2 \times \dot{Z}) \frac{\dot{y}}{y}+\dot{Z}=\frac{p_{t}}{c} \\
4 x Z \frac{\ddot{y}}{y}+\dot{Z}\left(1+2 x \frac{\dot{y}}{y}\right)+\frac{1-Z}{x}=\frac{\Delta}{c}
\end{gathered}
$$

where $\Delta=p_{t}-p_{r}$ is the measure of anisotropy and dots denote differentiation with respect to $\mathrm{x}$.

With the transformations of Durgapal and Bannerji [47], the mass within a radius $r$ of the sphere takes the form

$$
M(x)=\frac{1}{4 c^{3 / 2}} \int_{0}^{x} \sqrt{x} \rho(x) d x
$$

In this paper, the equation of state for radial pressure is presented in the form

$$
p_{r}=\alpha \rho+\beta \rho^{1 / 2}-\gamma
$$

proposed by Rocha et al.[41]. In eq. (10) $\alpha, \beta$ and $\gamma$ are arbitrary constants and $\rho$ is the energy density.

\section{Classes of Models}

In this treatment, we have chosen the form of the 
gravitational potential as $Z(x)=(1-\eta a x)^{2}$ where is $a$ real constant and $\eta$ is an adjustable parameter. This potential is well behaved and regular at the origin in the interior of the sphere. We have considered the particular cases for $\eta=3 / 2,3$.

For the case $\eta=3 / 2$, using $Z(x)$ in eq.(5), we obtain

$$
\begin{gathered}
\rho=3 a c\left(3-\frac{15}{4} a x\right) \quad M(x)=\frac{3 a(4-3 a x)}{8 \sqrt{c}} x^{3 / 2} \\
\text { With (11) and (12), eq. (6) becomes } \\
\frac{\dot{y}}{y}=\frac{3 a a\left(3-\frac{15}{4} a x\right)}{4\left(1-\frac{3}{2} a x\right)^{2}}+\frac{\beta\left[3 a c\left(3-\frac{15}{4} a x\right)\right]^{1 / 2}}{4 c\left(1-\frac{3}{2} a x\right)^{2}}-\frac{\gamma}{4 c\left(1-\frac{3}{2} a x\right)^{2}}+\frac{3 a-\frac{9}{4} a^{2} x}{4\left(1-\frac{3}{2} a x\right)^{2}}
\end{gathered}
$$
be written in the form
Substituting (11) into eq. (10), the radial pressure can

$$
p_{r}=3 \alpha a c\left(3-\frac{15}{4} a x\right)+\beta\left[3 a c\left(3-\frac{15}{4} a x\right)\right]^{1 / 2}-\gamma
$$

Using (11) in (9), the expression of the mass function is

Integrating (14), we have

$$
y(x)=c_{1}(3 a x-2)^{A^{*}} e^{\frac{5 \beta \sqrt{6} a c(3 a x-2) \arctan \left(\frac{1}{2} \sqrt{\frac{-30 a^{2} c x+24 a c}{a c}}\right)-6 \beta \sqrt{\left(-5 a^{2} c x+4 a c\right) a c}+B}{12 a c(3 a x-2) \sqrt{a c}}}
$$

where for convenience we have let

$$
A^{*}=-\frac{5}{4} \alpha-\frac{1}{4} \text { and } B=-6 \alpha a c \sqrt{a c}-6 a c \sqrt{a c}+4 \gamma \sqrt{a c}
$$

$\mathcal{C}_{1}$ is the constant of integration.

The anisotropy factor $\Delta$ is given by

$$
\Delta=4 x c\left(1-\frac{3}{2} a x\right)^{2} \frac{\ddot{y}}{y}-3 a c\left(1-\frac{3}{2} a x\right)\left(1+2 x \frac{\dot{y}}{y}\right)+3 a c-\frac{9}{4} a^{2} c x
$$

The metric functions $e^{2 \lambda}$ and $e^{2 v}$ can be written as

$$
\begin{gathered}
e^{2 \lambda}=\frac{1}{\left(1-\frac{3}{2} a x\right)^{2}} \\
e^{2 v}=A^{2} c_{1}^{2}(3 a x-2)^{2 A^{*}} e^{\frac{5 \beta \sqrt{6} a c(3 a x-2) \arctan \left(\frac{1}{2} \sqrt{\frac{-30 a^{2} c x+24 a c}{a c}}\right)-6 \beta \sqrt{\left(-5 a^{2} c x+4 a c\right) a c}+B}{6 a c(3 a x-2) \sqrt{a c}}}
\end{gathered}
$$

With $\eta=3$, the expression for the energy density is

$$
\rho=9 a c(2-5 a x)
$$

Replacing (19) in (10), we have the radial pressure as

$$
p_{r}=9 \alpha a c(2-5 a x)+\beta[9 a c(3-5 a x)]^{1 / 2}-\gamma
$$


and the mass function is

$$
M(x)=\frac{3 a(2-3 a x)}{2 \sqrt{c}} x^{3 / 2}
$$

With (19) and (20), the eq. (6) can be written as

$$
\frac{\dot{y}}{y}=\frac{9 \alpha a(2-5 a x)}{4(1-3 a x)^{2}}+\frac{\beta[9 a c(2-5 a x)]^{1 / 2}}{4 c(1-3 a x)^{2}}-\frac{\gamma}{4 c(1-3 a x)^{2}}+\frac{6 a-9 a^{2} x}{4(1-3 a x)^{2}}
$$

Integrating (22), we obtain

$$
y(x)=c_{2}(3 a x-1)^{A^{*}} e^{\frac{5 \beta \sqrt{3} a c(3 a x-1) \arctan \left(\frac{1}{2} \sqrt{\frac{-15 a^{2} c x+6 a c}{a c}}\right)-3 \beta \sqrt{\left(-5 a^{2} c x+2 a c\right) a c}+C}{12 a c(3 a x-1) \sqrt{a c}}}
$$

Again, for convenience we have let $C=-3 \alpha a c \sqrt{a c}-3 a c \sqrt{a c}+\gamma \sqrt{a c}$ and $C_{2}$ is the constant of integration.

The metric functions $e^{2 \lambda}, e^{2 v}$ and the anisotropy factor $\Delta$ can be written as

$$
\begin{gathered}
e^{2 \lambda}=\frac{1}{(1-3 a x)^{2}} \\
e^{2 v}=A^{2} c_{2}^{2}(3 a x-1)^{2 A^{*}} e^{\frac{5 \beta \sqrt{3} a c(3 a x-1) \arctan \left(\frac{1}{2} \sqrt{\frac{-15 a^{2} c x+6 a c}{a c}}\right)-3 \beta \sqrt{\left(-5 a^{2} c x+2 a c\right) a c+C}}{6 a c(3 a x-1) \sqrt{a c}}} \\
\Delta=4 x c(1-3 a x)^{2} \frac{\ddot{y}}{y}-6 a c(1-3 a x)\left(1+2 x \frac{\dot{y}}{y}\right)+6 a c-9 a^{2} c x
\end{gathered}
$$

\section{Physical Acceptability Conditions}

For a solution of the field equations to be physically acceptable [10, 48, 49], they must satisfy in the following conditions:

i) Regularity of the gravitational potentials in the stellar interior and at the origin.

ii) The radial pressure should be positive and a decreasing function of radial coordinate.

iii) The energy density should be well defined, positive and a decreasing function of the radial parameter.

iv) $\quad p_{r}>0$ and $\rho>0$ in the origin.

v) Any physically acceptable solution must satisfy the causality condition where the radial speed of sound should be less than speed of light throughout the model, i.e $0 \leq \frac{d p_{r}}{d \rho} \leq 1$.

vi) For the anisotropic case, the radial and the tangential pressure are equal to zero at the centre $r=0$, i.e. $\Delta(r=0)=0$. vii) In the surface of the sphere, it should be matched with the Schwarzschild exterior solution, for which the metric is given by

$$
d s^{2}=-\left(1-\frac{2 M}{r}\right) d t^{2}+\left(1-\frac{2 M}{r}\right)^{-1} d r^{2}+r^{2}\left(d \theta^{2}+\sin ^{2} d \phi^{2}\right)
$$

through the boundary $r=R$ where $M$ is the total mass of the star.

\section{Physical Analysis of the New Models}

For the case $\eta=3 / 2$, we have $e^{2 \lambda(0)}=1$ $e^{2 v(0)}=A^{2} c_{1}^{2}(-2)^{2 A^{*}} e^{\frac{-10 \beta a c \sqrt{6} \arctan (\sqrt{6})-12 \beta a c+B}{-24 a c \sqrt{a c}}}$ in the origin $r=0 \quad$ and $\left(e^{2 \lambda(r)}\right)_{r=0}^{\prime}=\left(e^{2 v(r)}\right)_{r=0}^{\prime}=0$. This shows that the potential gravitational is regular in the origin. In the centre $r \quad=0, \quad \rho(0)=9 \alpha a c \quad$ and 
$p_{r}(0)=9 \alpha a c+\beta \sqrt{3 a c}-\gamma$, both are positive if $a, \alpha, \beta, \gamma>0$. In the surface of the star $r=R$, we

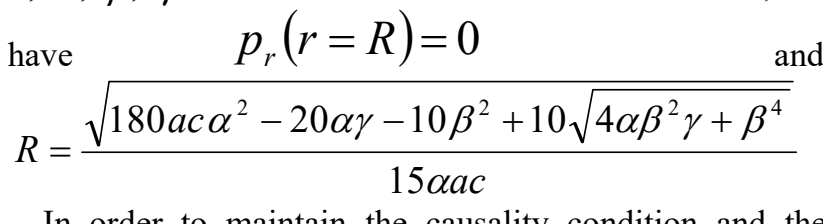

In order to maintain the causality condition and the regularity of metric potentials, they have been chosen for $\alpha, \beta$ and $\gamma$ the values $\alpha=1 / 5, \beta=1 / 10$ and $\gamma=0$. Then $R$ takes the form $R=\frac{2}{5} \sqrt{\frac{5}{a c}}$. For the pressure and density gradients for all $0<r<R$, we obtain respectively

$$
\frac{d \rho}{d r}=-\frac{45}{2} a^{2} c^{2} r<0
$$

$$
\frac{d p_{r}}{d r}=-\frac{45}{2} \alpha a^{2} c^{2} r-\frac{45 \beta a^{2} c^{2} r}{4 \sqrt{3 a c\left(3-\frac{15}{4} a c r^{2}\right)}}<0
$$

and according to the equations (28) and (29) the energy density and radial pressure decrease from the centre to the surface of the star.

From (13), we have

$$
M(r)=\frac{3 a c r^{3}\left(4-3 a c r^{2}\right)}{8}
$$

and the total mass of the star is

$$
M(r=R)=\frac{\left(180 a c \alpha^{2}-20 \alpha \gamma-10 \beta^{2}+10 \sqrt{4 \alpha \beta^{2} \gamma+\beta^{4}}\right)^{3 / 2}\left(12 a c \alpha^{2}+2 \alpha \gamma+\beta^{2}-\sqrt{4 \alpha \beta^{2} \gamma+\beta^{4}}\right)}{67500 \alpha^{5} a^{3} c^{3}}
$$

If $\alpha=1 / 5, \beta=1 / 10$ and $\gamma=0$, the eq. (31) take the form $M(r=R)=\frac{24}{125} \sqrt{\frac{5}{a c}}$

Matching conditions for $\mathrm{r}=\mathrm{R}$ can be written as

$$
\begin{gathered}
\left(1-\frac{2 M}{R}\right)=A^{2} y^{2}\left(c r^{2}\right) \\
\left(1-\frac{2 M}{R}\right)^{-1}=\frac{1}{\left(1-\frac{3}{2} a c R^{2}\right)^{2}}
\end{gathered}
$$

In order to maintain of causality, the radial sound speed defined as $v_{s r}^{2}=\frac{d p_{r}}{d \rho}$ should be within the limit $0 \leq v_{s r}^{2} \leq 1$ in the interior of the star [4]. In this model, we have:

$$
v_{s r}^{2}=\frac{d p_{r}}{d \rho}=\alpha+\frac{\beta}{2 \sqrt{3 a c\left(3-\frac{15}{4} a x\right)}}
$$

and for the eq.(34) we can impose the condition

$$
0 \leq \frac{\beta+2 \alpha \sqrt{3 a c\left(3-\frac{15}{4} a c r^{2}\right)}}{2 \sqrt{3 a c\left(3-\frac{15}{4} a c r^{2}\right)}} \leq 1
$$

With $\eta=3$, we have $e^{2 \lambda(0)}=1, e^{2 v(0)}=A^{2} c_{2}^{2}(-1)^{2 A^{*}} e^{\frac{5 \beta a c \sqrt{3} \arctan \left(\frac{\sqrt{6}}{2}\right)+3 \beta a c \sqrt{2}-C}{6 a c \sqrt{a c}}}$

With $\eta=3$, we have $e^{2 \lambda(0)}=1, e^{2 v(0)}=A^{2} c_{2}^{2}(-1)^{2 A^{*}} e^{6 a c \sqrt{a c}} \quad$ in the origin and 
$\left(e^{2 \lambda(r)}\right)_{r=0}^{\prime}=\left(e^{2 v(r)}\right)_{r=0}^{\prime}=0$. Again the gravitational potential is regular in $r=0$.

In the centre $\rho(0)=18 a c$ and $p_{r}(0)=18 \alpha a c+3 \beta \sqrt{a c}-\gamma$, both are positive if $a, \alpha, \beta, \gamma>0$. In the boundary of the star $r=R$, we have $p_{r}(r=R)=0$ and $R=\frac{\sqrt{360 a c \alpha^{2}-20 \alpha \gamma-10 \beta^{2}+10 \sqrt{4 \alpha \beta^{2} \gamma+\beta^{4}}}}{30 \alpha a c}$. If $\alpha=1 / 5, \beta=1 / 10$ and $\gamma=0$, then we obtain for the stellar radius $R=\frac{1}{5} \sqrt{\frac{10}{a c}}$. This is a new value found for the radius of the star.

As the radial pressure and the energy density decrease from the centre to the surface of the star we have that for all 0 $<r<R$

$$
\begin{gathered}
\frac{d \rho}{d r}=-90 a^{2} c^{2} r<0 \\
\frac{d p_{r}}{d r}=-90 \alpha a^{2} c^{2} r-\frac{15 \beta a^{2} c^{2} r}{\sqrt{a c\left(3-5 a c r^{2}\right)}}<0
\end{gathered}
$$

From (21),we get

$$
M(r)=\frac{3 a c r^{3}\left(2-3 a c r^{2}\right)}{2}
$$

and the total mass of the star is

$$
M(r=R)=\frac{\left(360 a c \alpha^{2}-20 \alpha \gamma-10 \beta^{2}+10 \sqrt{4 \alpha \beta^{2} \gamma+\beta^{4}}\right)^{3 / 2}\left(24 a c \alpha^{2}+2 \alpha \gamma+\beta^{2}-\sqrt{4 \alpha \beta^{2} \gamma+\beta^{4}}\right)}{540000 \alpha^{5} a^{3} c^{3}}
$$

If $\alpha=1 / 5, \beta=1 / 10$ and $\gamma=0$, the eq. (39) takes the form $M(r=R)=\frac{12}{125} \sqrt{\frac{10}{a c}}$.

Matching conditions for $r=R$ can be written as

$$
\left(1-\frac{2 M}{R}\right)=A^{2} y^{2}\left(c r^{2}\right) \text { and }\left(1-\frac{2 M}{R}\right)^{-1}=\frac{1}{\left(1-3 a c R^{2}\right)^{2}}
$$

For this case, the condition $0 \leq v_{s r}^{2} \leq 1$, also implies that

$$
0 \leq \frac{\beta+2 \alpha \sqrt{9 a c\left(2-5 a c r^{2}\right)}}{2 \sqrt{9 a c\left(2-5 a c r^{2}\right)}} \leq 1
$$

The figures 1,2,3,4 and 5 represent the graphs of $p_{r}, \rho, M(x), \Delta$ and $v_{s r}^{2}$, respectively with $\eta=3 / 2, \alpha=1 / 5, \beta=1 / 10$, $\gamma=0, a=0.028$ and a stellar radius of $r=5.3 \mathrm{~km}$. 


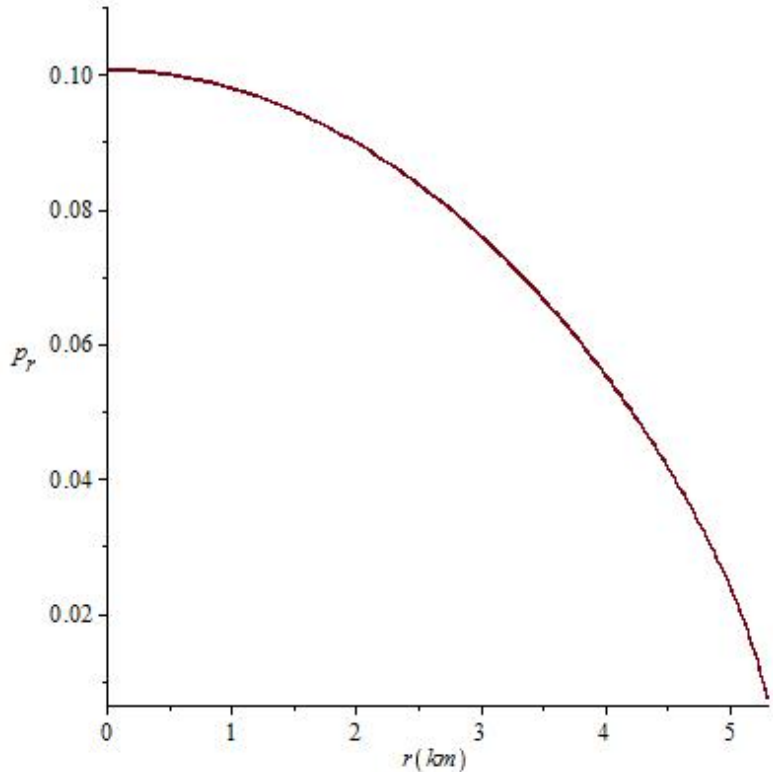

Figure 1. Radial pressure vs radial coordinate for $\eta=3 / 2, \alpha=1 / 5$, $\beta=1 / 10, \gamma=0$ where $a=0.028$ and $c=1$

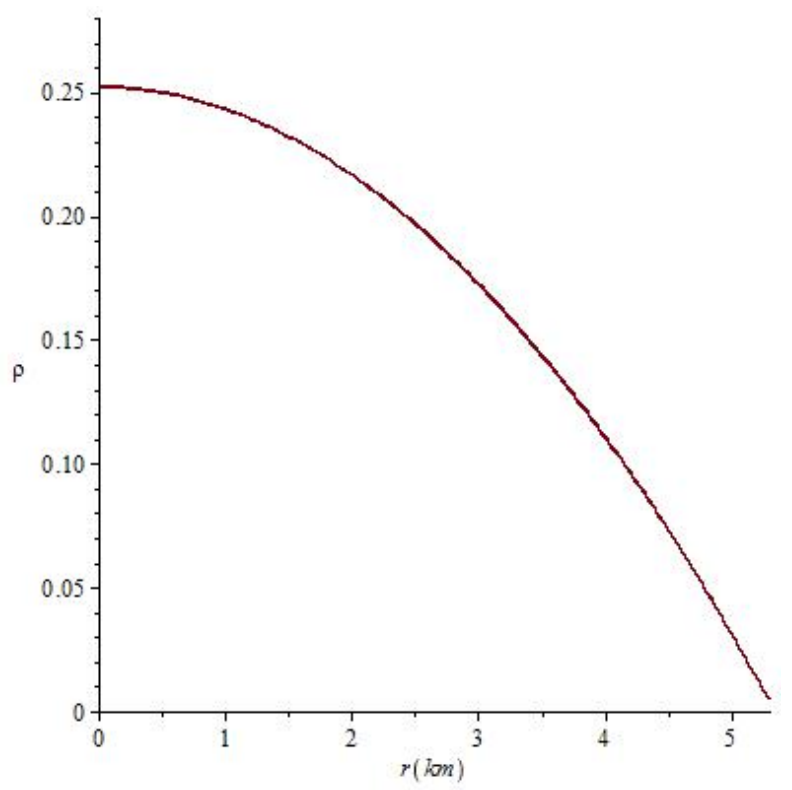

Figure 2. Energy density vs radial coordinate for $\eta=3 / 2, \alpha=1 / 5, \beta=1 / 10$, $\gamma=0$ where $a=0.028$ and $c=1$

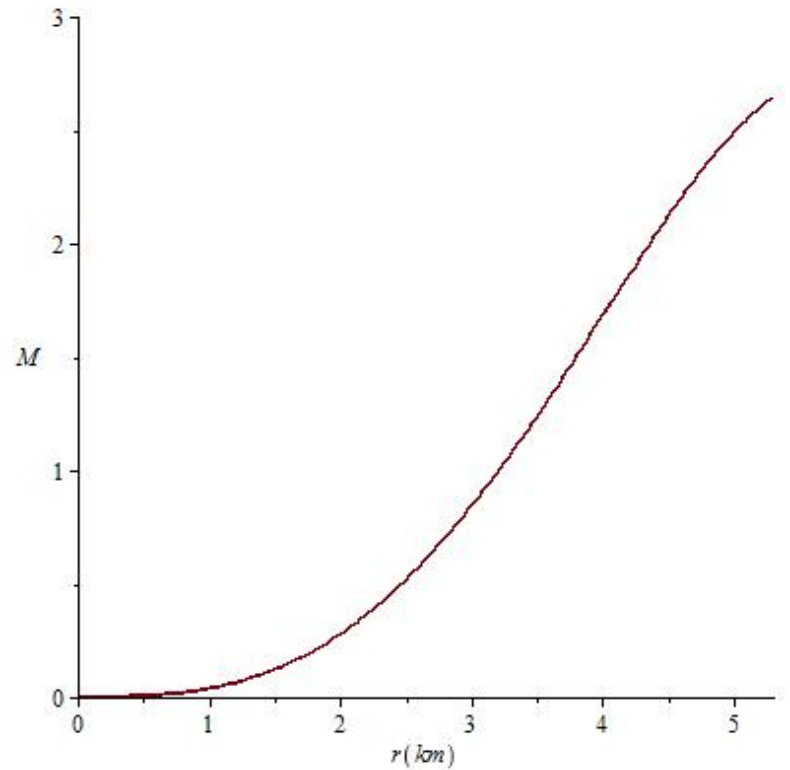

Figure 3. Mass function vs radial coordinate for for $\eta=3 / 2, \alpha=1 / 5$, $\beta=1 / 10, \gamma=0$ where $a=0.028$ and $c=1$

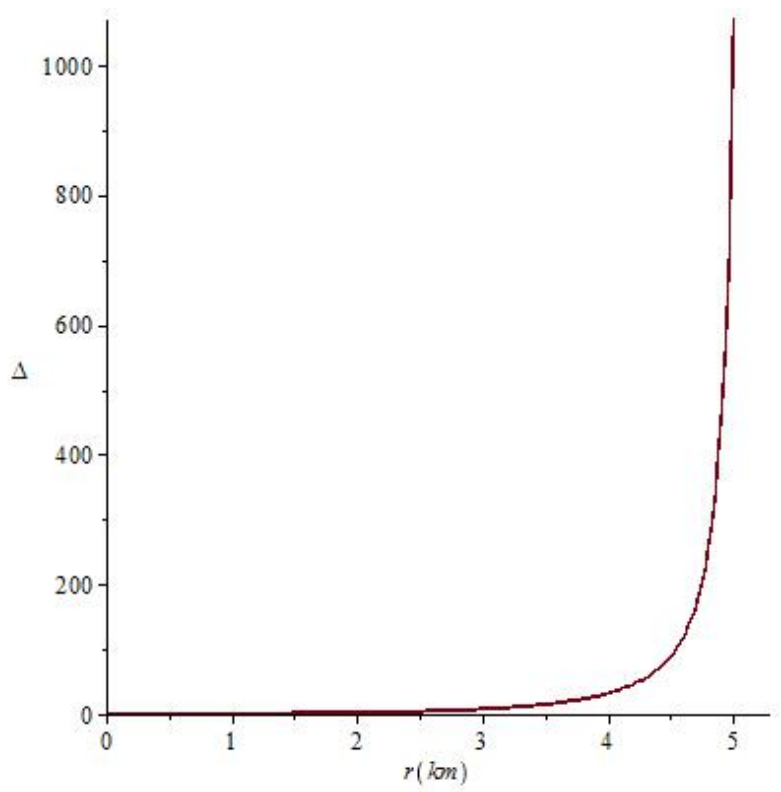

Figure 4. Measure of anisotropy vs radial coordinate for $\eta=3 / 2, \alpha=1 / 5$, $\beta=1 / 10, \gamma=0$ where $a=0.028$ and $c=1$ 


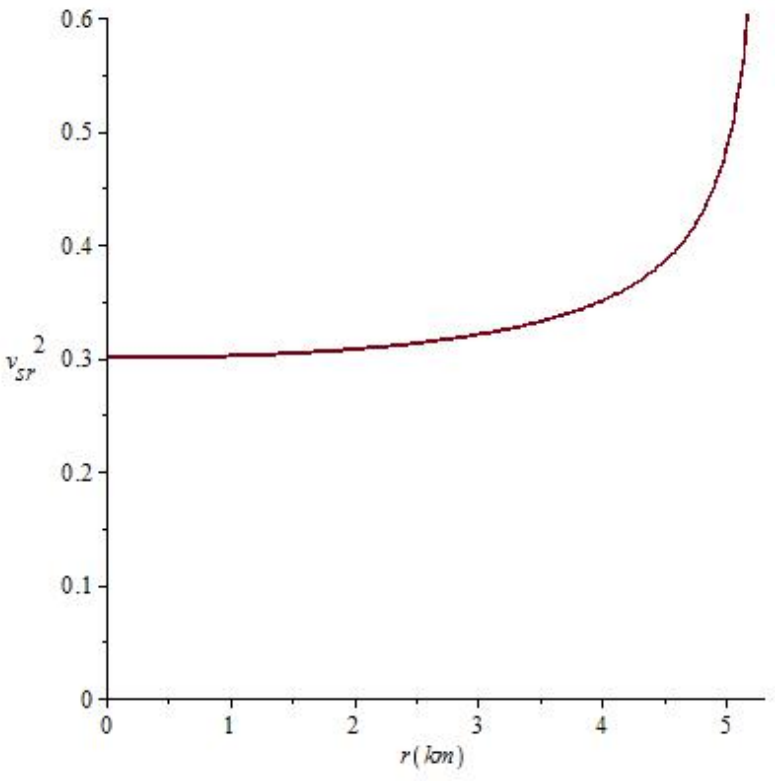

Figure 5. Radial speed sound vs radial coordinate for $\eta=3 / 2$, $\alpha=1 / 5, \beta=1 / 10, \gamma=0$ where $a=0.028$ and $c=1$

In figure 1 , it is observed that the radial pressure is finite and decreasing from the center to the surface of the star. In figure 2, the energy density is continuous, also is finite and monotonically decreasing function. In figure 3, the mass function is strictly increasing, continuous and finite. In figure 4 , the measure of anisotropy is increasing and continuous in the stellar interior and $\Delta$ vanishes at the center and this means that the radial and tangential pressures should be equal in $r=0$. The figure 5 shows that the condition $0 \leq v^{2}{ }_{s r} \leq 1$ is maintained throughout the interior of the star and satisfy the causality, which is a physical requirement for the construction of a realistic star [4].

\section{Conclusions}

In this research, we have generated some new class of exact models with an equation of state that considers CFL strange matter phase and anisotropy in the pressure where the gravitational potential $Z$ depends on an adjustable parameter $\eta$. All the obtained models are physically reasonable and satisfy the physical characteristics of a realistic star as are the regularity of the gravitational potentials at the origin, cancellation of anisotropy in $r=0$, radial pressure finite at the centre and decreasing of the energy density and the radial pressure from the centre to the surface of the star. These solutions match with the Schwarzschild exterior metric at the boundary for each value of adjustable parameter and the CFL phase is modelled, as it is electrically neutral according to Rocha et al. [41].

The values calculated for energy density, mass and stellar radius could correspond to compact objects with real existence. For $\eta=3$, the radius and total mass of the star is given by $R=\frac{1}{5} \sqrt{\frac{10}{a c}}$ and $M(r=R)=\frac{12}{125} \sqrt{\frac{10}{a c}}$ with $\alpha=1 / 5, \beta=1 / 10$ and $\gamma=0$. When $\eta=3 / 2, \quad R=\frac{1}{5} \sqrt{\frac{10}{a c}}$ and $M(r=R)=\frac{12}{125} \sqrt{\frac{10}{a c}}$. We can then generate models with anisotropy in the pressure made of CFL strange matter with defined values of mass and radius. The values of $\alpha, \beta$ and $\gamma$ have been chosen in order to maintain the causality condition and the regularity of metric potentials inside the radius of the star.

With the CFL equation of state, the MIT bag model can be recovered as a particular case of this work by taking $\beta=0$ in eq. (10) and generates families of exact solutions for the Einstein-Maxwell field equations for modeling relativistic compact objects, strange stars and configurations with anisotropic matter distribution.

\section{REFERENCES}

[1] Kuhfitting, P.K.(2011). Some remarks on exact wormhole solutions, Adv. Stud. Theor. Phys., 5, 365-367.

[2] Bicak, J.(2006). Einstein equations: exact solutions, Encyclopaedia of Mathematical Physics, 2, 165-173.

[3] Malaver, M. (2013). Black Holes, Wormholes and Dark Energy Stars in General Relativity. Lambert Academic Publishing, Berlin. ISBN: 978-3-659-34784-9.

[4] Delgaty, M.S.R and Lake, K. (1998). Physical Acceptability of Isolated, Static, Spherically Symmetric, Perfect Fluid Solutions of Einstein's Equations, Comput. Phys. Commun. 115, 395-415.

[5] Schwarzschild, K. (1916). Uber das Gravitationsfeld einer Kugel aus inkompressibler Flussigkeit nach der Einsteinschen Theorie. Math.Phys.Tech, 424-434.

[6] Tolman, R.C.(1939). Static Solutions of Einstein's Field Equations for Spheres of Fluid. Phys. Rev., 55(4), 364-373.

[7] Oppenheimer, J.R. and Volkoff, G. (1939). On Massive Neutron Cores. Phys. Rev., 55(4), 374-381.

[8] Chandrasekahr, S. (1931). The Maximum Mass of Ideal White Dwarfs. Astrophys. J, 74, 81-82.

[9] Baade, W. and Zwicky, F. (1934). On Super-novae. Proc.Nat.Acad.Sci.U.S 20(5), 254-259.

[10] Thirukkanesh, S. and Ragel, F.C. (2012). Exact anisotropic sphere with polytropic equation of state, PRAMANA-Journal of physics, 78(5), 687-696.

[11] Itoh, N. (1970). Hydrostatic equilibrium of hypothetical quark stars, Prog. Theor. Phys. 44, 291-292. 
[12] Komathiraj, K., and Maharaj, S.D. (2007). Analytical models for quark stars. Int. J. Mod. Phys. D16, 1803-1811.

[13] Cosenza, M., Herrera, L., Esculpi, M. and Witten, L. (1981). Some Models of Anisotropic Spheres in General Relativity, J. Math. Phys, 22(1), 118.

[14] Gokhroo, M. K. and Mehra, A. L. (1994). Anisotropic Spheres with Variable Energy Density in General Relativity. Gen. Relat. Grav, 26(1), 75 -84.

[15] Esculpi, M., Malaver, M. and Aloma, E. (2007). A Comparative Analysis of the Adiabatic Stability of Anisotropic Spherically Symmetric solutions in General Relativity. Gen. Relat. Grav, 39(5), 633-652.

[16] Malaver, M. (2018). Generalized Nonsingular Model for Compact Stars Electrically Charged. World Scientific News, 92(2), 327-339.

[17] Malaver, M. (2018). Some new models of anisotropic compact stars with quadratic equation of state. World Scientific News, 109, 180-194.

[18] Chan R., Herrera L. and Santos N. O.(1992). Dynamical instability in the collapse of anisotropic matter. Class. Quantum Grav, 9(10), L133.

[19] Malaver, M. (2017). New Mathematical Models of Compact Stars with Charge Distributions. International Journal of Systems Science and Applied Mathematics, 2(5), 93-98.

[20] Cosenza M., Herrera L., Esculpi M. and Witten L.(1982). Evolution of radiating anisotropic spheres in general relativity. Phys.Rev. D, 25(10), 2527-2535.

[21] Herrera L.(1992). Cracking of self-gravitating compact objects. Phys. Lett. A, 165, 206-210.

[22] Herrera L. and Ponce de Leon J.(1985). Perfect fluid spheres admitting a one - parameter group of conformal motions. J.Math.Phys, 26, 778.

[23] Herrera L. and Nunez L.(1989). Modeling 'hydrodynamic phase transitions' in a radiating spherically symmetric distribution of matter. The Astrophysical Journal, 339(1), 339-353.

[24] Herrera L., Ruggeri G. J. and Witten L.(1979). Adiabatic Contraction of Anisotropic Spheres in General Relativity. The Astrophysical Journal, 234, 1094-1099.

[25] Herrera L., Jimenez L., Leal L., Ponce de Leon J., Esculpi $\mathrm{M}$ and Galina V.(1984). Anisotropic fluids and conformal motions in general relativity. J. Math. Phys, 25, 3274.

[26] Sokolov. A. I. (1980). Phase transitions in a superfluid neutron liquid. Sov. Phys. JETP, 52(4), 575-576.

[27] Usov, V. V. (2004). Electric fields at the quark surface of strange stars in the color- flavor locked phase. Phys. Rev. D, 70(6), 067301.

[28] Komathiraj, K. and Maharaj,S.D. (2008). Classes of exact Einstein-Maxwell solutions,Gen. Rel.Grav. 39(12), 2079-2093.

[29] Thirukkanesh, S. and Maharaj, S.D.(2008). Charged anisotropic matter with a linear equation of state. Class. Quantum Gravity, 25(23), 235001.
[30] Maharaj, S.D., Sunzu, J.M. and Ray, S. (2014). Some simple models for quark stars. Eur. Phys. J.Plus, 129, 3.

[31] Thirukkanesh, S. and Ragel, F.C.(2013). A class of exact strange quark star model. PRAMANA-Journal of physics, 81(2), 275-286.

[32] Thirukkanesh, S. and Ragel, F.C. (2014). Strange star model with Tolmann IV type potential, Astrophysics and Space Science, 352(2), 743-749.

[33] Feroze, T. and Siddiqui, A. (2011). Charged anisotropic matter with quadratic equation of state. Gen. Rel. Grav, 43, 1025-1035.

[34] Feroze, T. and Siddiqui, A. (2014). Some Exact Solutions of the Einstein-Maxwell Equations with a Quadratic Equation of State. Journal of the Korean Physical Society, 65(6), 944-947.

[35] Sunzu, J.M, Maharaj, S.D. and Ray, S.(2014). Quark star model with charged anisotropic matter. Astrophysics. Space.Sci, 354, 517-524.

[36] Pant, N., Pradhan, N. and Malaver, M.(2015). Anisotropic fluid star model in isotropic coordinates. International Journal of Astrophysics and Space Science. Special Issue: Compact Objects in General Relativity. 3(1), 1-5.

[37] Malaver, M.(2014). Strange Quark Star Model with Quadratic Equation of State. Frontiers of Mathematics and Its Applications, 1(1), 9-15.

[38] Malaver, M. (2018).Charged anisotropic models in a modified Tolman IV space time. World Scientific News, $101,31-43$.

[39] Malaver, M. (2018). Charged stellar model with a prescribed form of metric function $\mathrm{y}(\mathrm{x})$ in a Tolman VII spacetime. World Scientific News, 108, 41-52.

[40] Malaver, M. (2016). Classes of relativistic stars with quadratic equation of state. World Scientific News, 57, 70 -80 .

[41] Rocha, L.S., Bernardo, A., De Avellar, M.G.B. and Horvath, J.E. (2019). Exact solutions for compact stars with CFL quark matter. Arxiv: 1906.11311.

[42] Malaver, M. (2009). Análisis comparativo de algunos modelos analíticos para estrellas de quarks, Revista Integración, 27(2), 125-133.

[43] Bombaci, I. (1997). Observational evidence for strange matter in compact objects from the $\mathrm{x}$ - ray burster $4 \mathrm{U}$ 1820-30, Phys. Rev, C55, 1587- 1590.

[44] Dey, M., Bombaci, I, Dey, J, Ray, S and. Samanta, B.C.(1998). Strange stars with realistic quark vector interaction and phenomenological density-dependent scalar potential, Phys. Lett, B438, 123-128.

[45] Takisa, P.M. and Maharaj, S.D.(2013). Some charged polytropic models. Gen.Rel.Grav, 45, 1951-1969.

[46] Malaver, M. (2013). Analytical model for charged polytropic stars with Van der Waals Modified Equation of State, American Journal of Astronomy and Astrophysics, 1(4), 37-42. 
[47] Durgapal, M.C. and Bannerji, R.(1983). New analytical stellar model in general relativity. Phys.Rev. D27, 328-331.

[48] Bibi, R., Feroze, T. and Siddiqui, A. (2016). Solution of the Einstein-Maxwell Equations with Anisotropic Negative Pressure as a Potential Model of a Dark Energy Star. Canadian Journal of Physics, 94(8), 758-762.

[49] Bhar, P. and Murad,M.H. (2016). Relativistic compact anisotropic charged stellar models with Chaplygin equation of state. Astrophys. Space. Sci, 361(10), 334. 